\title{
Transient Au-CO Complexes Promote the Activity of an Inverse Ceria/Gold Catalyst: an Insight from Ab Initio Molecular Dynamics
}

Yong Li, ${ }^{1}$ Shikun Li, ${ }^{1}$ Marcus Bäumer, ${ }^{1}$ Lyudmila V. Moskaleva, ${ }^{1,2, *}$

${ }^{1}$ Institute of Applied and Physical Chemistry and Center for Environmental Research and Sustainable Technology, University of Bremen, 28359 Bremen, Germany

${ }^{2}$ Department of Chemistry, University of the Free State, PO Box 339, 9300 Bloemfontein, South Africa

* Corresponding author’s e-mail address: lyudmila.moskaleva@gmail.com 


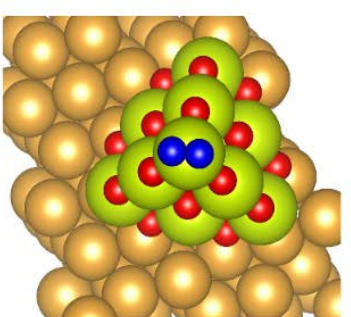

a)

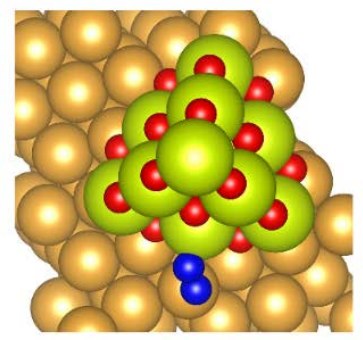

d)

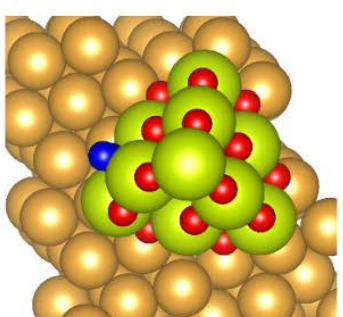

b)

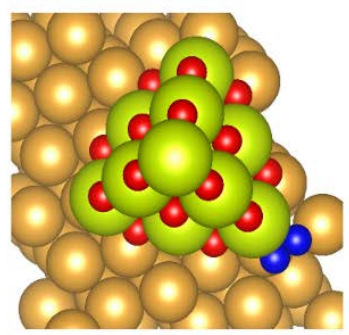

e)

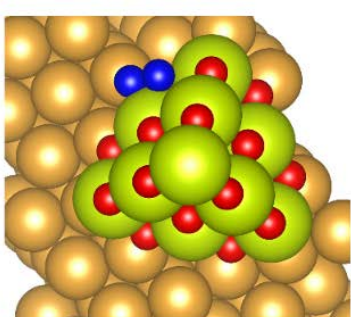

c)

Figure S1. Possible geometric configurations of $\mathrm{O}_{2}$ adsorption on the $\mathrm{Ce}_{10} \mathrm{O}_{19} / \mathrm{Au}(321)$ model. Color coding of elements: orange, $\mathrm{Au}$; lime, Ce; red, O; blue, adsorbed $\mathrm{O}_{2}$; gray, C.

Table S1. $\mathrm{O}_{2}$ adsorption on the $\mathrm{Ce}_{10} \mathrm{O}_{20 / 19} / \mathrm{Au}(321)$ models. Adsorption energies, spin population on $\mathrm{O}_{2}$, $\mathrm{O}-\mathrm{O}$ bond lengths.

\begin{tabular}{cccc}
\hline Configuration & $E_{\text {ads }}(\mathrm{eV})$ & Spin population & O-O bond length $(\AA)$ \\
a & -0.41 & $0.51,0.49$ & 1.37 \\
b & -0.64 & $0.51,0.49$ & 1.37 \\
c & -0.86 & $0.0,0.0$ & 1.44 \\
d & -0.66 & $0.10,0.13$ & 1.36 \\
e & -1.62 & $0.0,0.0$ & 1.47 \\
& & $\mathrm{Ce}_{10} \mathrm{O}_{20} / \mathrm{Au}(321)$ & \\
a & -0.11 & $101,0.97$ & 1.29 \\
b & -0.03 & $0.93,0.96$ & 1.27 \\
c & -0.31 & $0.58,0.71$ & 1.30 \\
d & -0.29 & $0.78,0.61$ & 1.30 \\
e & -1.57 & $0.0,0.0$ & 1.46 \\
\hline
\end{tabular}



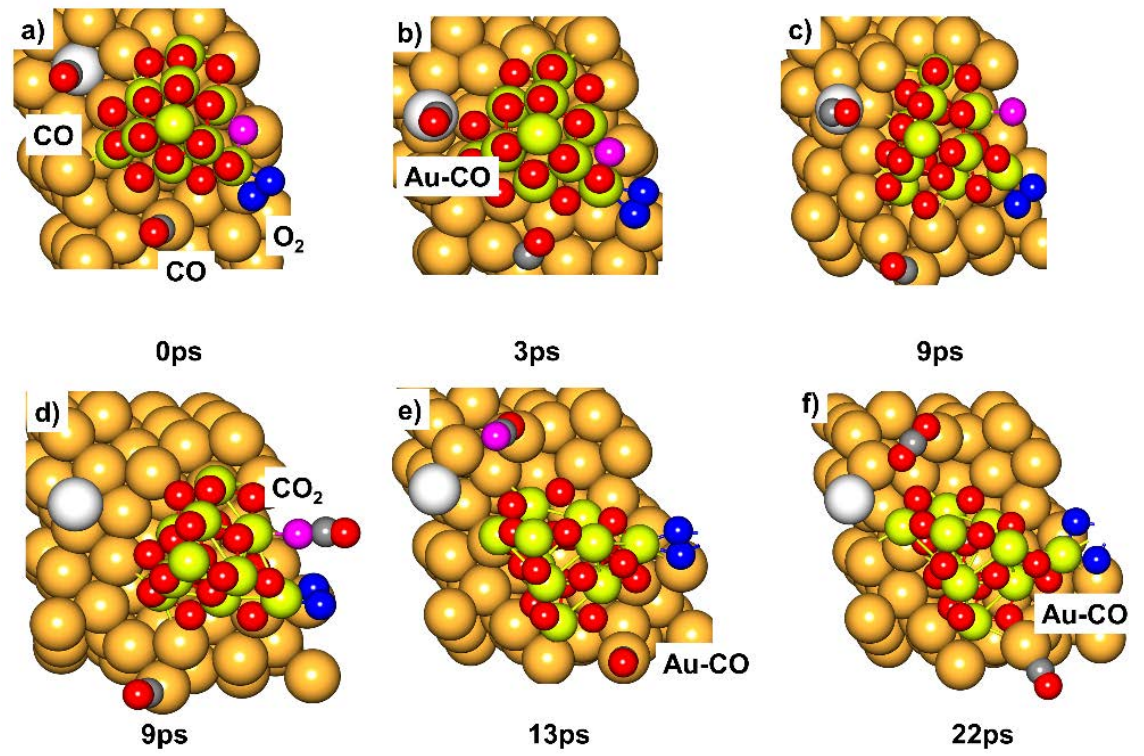

Figure S2. Snapshots from an AIMD simulation of $\mathrm{CO}$ oxidation on the $\mathrm{Ce}_{10} \mathrm{O}_{19} / \mathrm{Au}(321)$ model for $24 \mathrm{ps}$ at $700 \mathrm{~K}$. Color coding of elements: orange and white, $\mathrm{Au}$; lime, Ce; red and magenta, O; blue, adsorbed $\mathrm{O}_{2}$; gray, $\mathrm{C}$.

a)

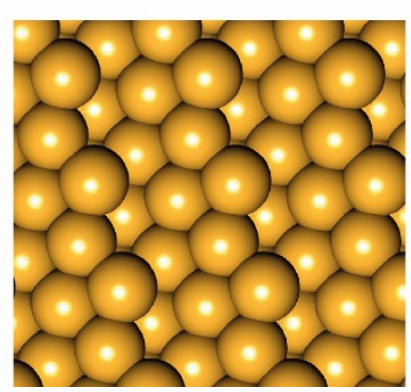

b)

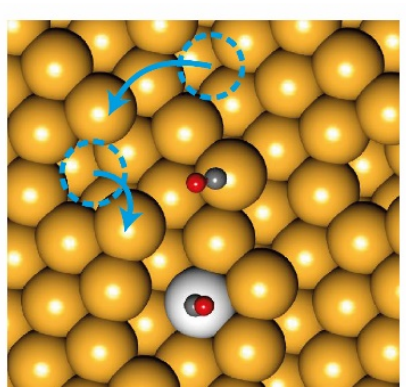

c)

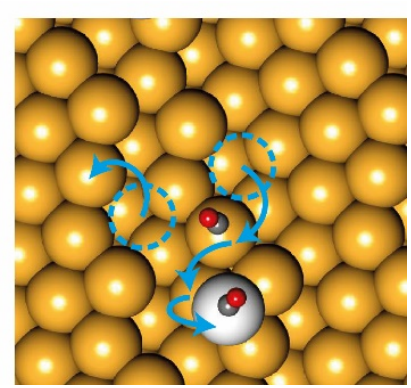

Figure S3. (a) Regular Au(321) surface. Initial (b) and final (c) states for the formation of Au-CO carbonyl on the Au surface with adsorbed $\mathrm{CO}$ with the $\mathrm{Ce}_{10} \mathrm{O}_{19}$ particle removed. White color marks the Au atom that is being extracted from the surface and is forming gold carbonyl. Blue dashed circles and arrows show which Au atoms have been displaced in (b) with respect to (a) and in (c) with respect to (b). 

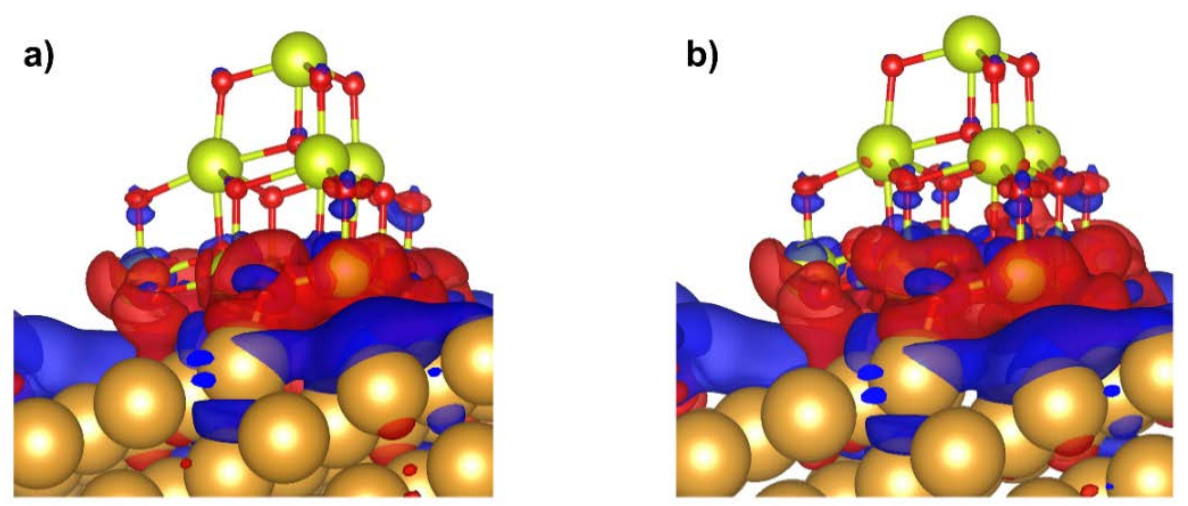

Figure S4. Charge density difference analysis for ceria nanoparticles supported on $\mathrm{Au}(321)$ : (a) perfect stoichiometric $\mathrm{Ce}_{10} \mathrm{O}_{20}$ model without a vacancy, (b) reduced $\mathrm{Ce}_{10} \mathrm{O}_{19}$ particle with an $\mathrm{O}$ vacancy at position 1 (see Figure 1c). The charge density difference was computed according to $\Delta \rho=\rho_{\mathrm{AB}}-\rho_{\mathrm{A}}-\rho_{\mathrm{B}}$, where A and B stand for a bare $\mathrm{Au}(321)$ surface and a free $\mathrm{Ce}_{10} \mathrm{O}_{19 / 20}$ cluster, respectively. Electron density increase is shown in blue, electron density decrease is shown in red. (Isovalue: 0.002 au.)

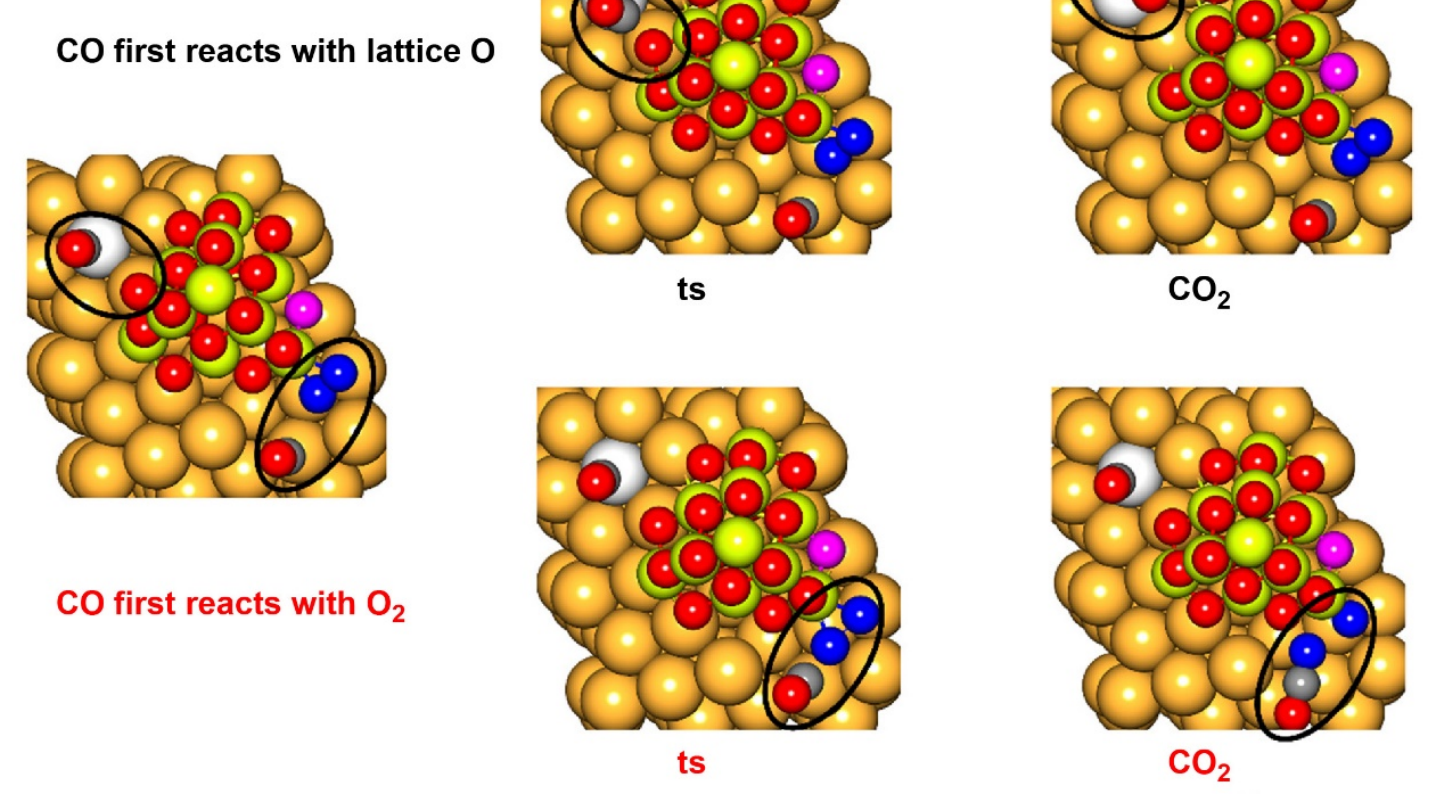

Figure S5. Initial structure, transition states and products corresponding to two higherenergy pathways for the first CO oxidation (first half of the cycle) shown by black and red bar diagrams in Figure 5 of the main article. The "black" and "red" pathways correspond to first $\mathrm{CO}$ being directly oxidized either by lattice $\mathrm{O}$ or by an $\mathrm{O}_{2}$ molecule, respectively. 


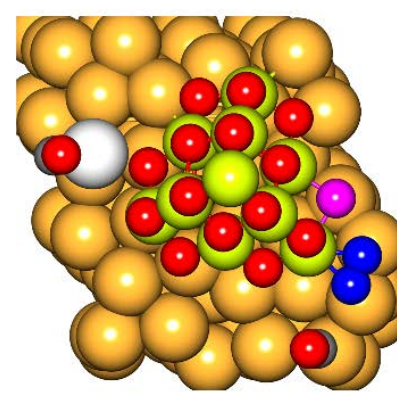

ts 1

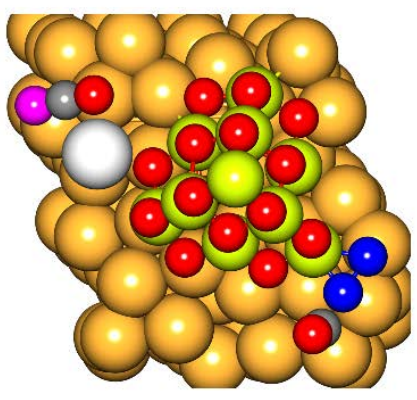

ts2

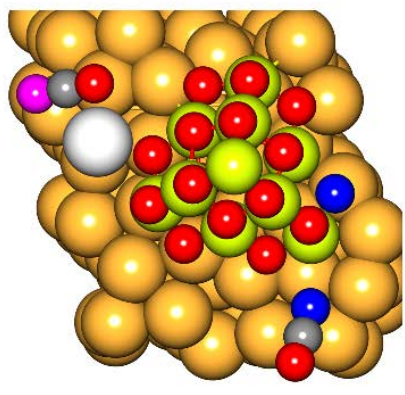

ts3

Figure S6. Transition state structures corresponding to the low-energy pathway associated with Au-CO formation shown by blue energy bar diagram in Figure 5 of the main article. 